\title{
A novel neuroimaging assessment of predictive risk factors associated with intraoperative massive hemorrhage in intracranial solid hemangioblastoma
}

\author{
Qiang Gao ${ }^{1}$, Lan Zhang ${ }^{2}$, Guoqing Wang ${ }^{1}$, Dingkang $\mathrm{Xu}^{1}$, Mengzhao Feng ${ }^{1}$, Fang Wang ${ }^{1}$, Qingjie Wei ${ }^{1}$, \\ Xianzhi Liu ${ }^{1}$, Fuyou Guo ${ }^{1}$ \\ ${ }^{1}$ Department of Neurosurgery, The First Affiliated Hospital of Zhengzhou University, Zhengzhou 450052, China; ${ }^{2}$ Department of MRI, The First \\ Affiliated Hospital of Henan University of Chinese Medicine, Zhengzhou 450003, China
}

Correspondence to: Fuyou Guo. Department of Neurosurgery, Key Laboratory of Neurosurgical Diseases, The First Affiliated Hospital of Zhengzhou University, Jianshe East Road, No. 1, Zhengzhou 450052, China. Email: chyou666@hotmail.com.

\begin{abstract}
Background: A solid hemangioblastoma (SH) is a benign and highly vascularized tumor. Microsurgical treatment of $\mathrm{SH}$ is still challenging due to excessive intraoperative bleeding.

Methods: Sixty-six consecutive patients with SH were retrospectively analyzed. The volume of intraoperative blood loss (IBL) and the features detected by magnetic resonance imaging (MRI) were evaluated by a neurosurgeon and multiple neuroradiologists.

Results: Four striking MRI features-peritumoral edema, the flow-void effect, large draining veins, and a visible feeding artery-were related to excessive IBL. Regarding the weighted values of these risk factors, the flow-void effect was the factor most significantly correlated with IBL $(\mathrm{P}<0.01, \mathrm{R}=0.418)$. The feeding artery also contributed substantially to excessive IBL $(\mathrm{P}<0.05, \mathrm{R}=0.412)$. The next most influential factor was the presence of large draining veins $(\mathrm{P}<0.05, \mathrm{R}=0.350)$, followed by peritumoral edema $(\mathrm{P}<0.05, \mathrm{R}=0.308)$. The four major risk factors - the flow-void effect, feeding arteries, large draining veins, and peritumoral edemawere assigned point values of 3,3,2, and 1, respectively, for a maximum total score of 9 points. A higher total score indicates that a higher volume of bleeding is more likely to occur during surgery.

Conclusions: This study reports the potential neuroimaging-based risk factors leading to abundant IBL in SH by neuroimaging assessment. The study proposes a novel scoring system to predict IBL, potentially decreasing the risk involved in the surgical treatment of SH.
\end{abstract}

Keywords: Solid hemangioblastomas (SHs); neuroimaging assessment; intraoperative blood loss (IBL); surgical risk prediction; neurosurgery

Submitted May 04, 2019. Accepted for publication Aug 30, 2019.

doi: 10.21037/qims.2019.09.05

View this article at: http://dx.doi.org/10.21037/qims.2019.09.05

\section{Introduction}

Hemangioblastomas are relatively rare true vascular tumors of the central nervous system, representing $1.5-2.5 \%$ of all intracranial tumors. Hemangioblastomas may occur sporadically $(75 \%)$ or in association with von Hippel-Lindau (VHL) disease (25\%) (1). Most tumors of this type occur on the dorsal surface of the brainstem and in the cerebellum. These growths often appear cystic and solid on magnetic resonance imaging (MRI). Solid hemangioblastomas (SHs) have an abundant blood supply and are found adjacent to important brain structures such as the brainstem, which makes it difficult to stop bleeding during surgery; intraoperative bleeding during $\mathrm{SH}$ treatment may lead to failure of the procedure or pose a risk to the patient's life postoperatively. Kuharic et al. (2) systematically analyzed 207 cases of 1,759 cases of large-scale vascular reticulocyte 
tumors. The total mortality rate of $\mathrm{SH}$ following surgery was $10.3 \%$, and the average intraoperative blood loss (IBL) was $675.85 \pm 470.29 \mathrm{~mL}$. In addition, Kageji et al. (3) reported that 1,149 cases of intracranial tumors had a hematoma mortality rate of $12.5 \%$ within 30 days after the first operation, and the average incidence rate of postoperative hematoma was $2.09 \%$. However, after hemangioblastoma treatment, the incidence of hematoma was much higher at $17.65 \%$; the authors believe that hemangioblastomas are closely associated with a significantly increased incidence of postoperative hematoma. Recently, Tabibkhooei et al. (4) reported that a patient with massive hemangioblastoma located in the cavernous sinus was forced to undergo subtotal resection due to severe catastrophic hemorrhage during surgery. The microsurgical removal of $\mathrm{SH}$ remains challenging because it is difficult to control intraoperative bleeding and postoperative complications.

How can neuroimaging be used to predict potential excessive bleeding in SH? To date, there has been no literature describing the prediction and analysis of excessive IBL in SH. Therefore, if risk factors that may cause excessive IBL in intracranial SH can be found by MRI, such knowledge would provide substantial benefits by improving the safety of surgery and reducing the risk of surgical complications. This study was conducted to analyze the neuroimaging-based risk factors associated with the amount of intraoperative $\mathrm{SH}$ bleeding and to establish a scoring system for the risk factors predicting the amount of intraoperative bleeding in this highly vascularized intracranial tumor. The greatest advantage of this study is that it will supply useful information from which to predict IBL, preparing surgeons to identify radiological features to create a preoperative complexity score for surgical planning.

\section{Methods}

\section{Patients and general data}

A total of 270 consecutive patients with hemangioblastomas who underwent microsurgical treatment at the First Affiliated Hospital of Zhengzhou University from January 2006 to January 2018 were reviewed systematically. Only 66 patients (42 men and 24 women) with SH were enrolled in this study according to the following eligibility criteria: (I) patients met the diagnostic criteria for $\mathrm{SH}$ (all cases were confirmed by definite pathology after surgery); (II) all operations were performed at our neurosurgical center; (III) only patients with complete medical records and followup information were included in this study; (IV) the MRI features of the $\mathrm{SH}$ patients had been analyzed independently by three senior neuroradiologists (all of whom were professors with at least 10 years of experience). The exclusion criteria consisted of cystic hemangioblastomas, incomplete preoperative data, and any intracranial tumor other than hemangioblastoma. The present study was approved by the ethics committee of Zhengzhou University in accordance with the Declaration of Helsinki. All patients gave written informed consent for the surgery and for the use of their data and samples for research purposes before the surgery. The baseline and clinical characteristics of all patients are summarized in Table 1. The patients' age at surgery ranged from 12 to 73 years (mean, 44.17 years). The mean duration between the first symptoms and diagnosis was 47.7 weeks (range, 0.43-520 weeks). Eight patients underwent preoperative embolization by guglielmi detachable coil (EV3, USA) and Clubran glue in the present study. Only 1 patient was diagnosed with VHL disease, and 65 patients were classified as having sporadic SH. Three patients had multiple lesions, and 63 patients harbored single lesions. The locations of the tumors are described in Table 1. Also, there were 12 patients whose preoperative MRI showed midline tumors misdiagnosed as medulloblastoma or ependymoma; a primary misdiagnosis of meningioma was given to 2 patients whose preoperative MRI showed tumors next to the tentorium.

\section{Neuroimaging and treatment}

All patients underwent tumor evaluation by MRI with intravenous administration of a contrast agent. Computed tomography (CT) and CT angiography (CTA) were conducted in 45 and 21 cases, respectively. Digital subtraction angiography (DSA) was adopted in 8 patients, and the staining of the tumors was remarkably diminished after preoperative embolization. Microsurgical treatment was performed via a midline suboccipital approach or a suboccipital retrosigmoid approach. Fifty-seven patients were followed up from 3 to 96 months, with an average of 48 months; 1 patient experienced tumor recurrence during follow-up, the remaining 9 patients were lost to follow-up.

\section{Search strategy}

All patient information in the medical and surgical records 
Table 1 Summary of general characteristics in patients with SH

\begin{tabular}{|c|c|}
\hline Characteristics & Value \\
\hline \multicolumn{2}{|l|}{ Age, years } \\
\hline Mean \pm SD & $44.17 \pm 12.75$ \\
\hline Range & $12-73$ \\
\hline \multicolumn{2}{|l|}{ Sex, n [\%] } \\
\hline Male & 42 [64] \\
\hline Female & 24 [36] \\
\hline Mean diameter $\pm \mathrm{SD}, \mathrm{mm}$ & $38.20 \pm 12.47$ \\
\hline Preop embolization, n [\%] & $8[12]$ \\
\hline Recurrence, $\mathrm{n}[\%]$ & $1[2]$ \\
\hline VHL, n [\%] & $1[2]$ \\
\hline Surgery, n [\%] & $66[100]$ \\
\hline Mean op time $\pm S D, h$ & $3.58 \pm 1.26$ \\
\hline \multicolumn{2}{|l|}{ Location of tumor, n [\%] } \\
\hline Posterior fossa & $3[5]$ \\
\hline Brainstem & $1[2]$ \\
\hline Fourth ventricle & $7[11]$ \\
\hline Vermis & $5[8]$ \\
\hline Medulla & $4[6]$ \\
\hline Peduncles & $2[3]$ \\
\hline Right cerebellum & 20 [30] \\
\hline Left cerebellum & $21[32]$ \\
\hline Multiple & $3[5]$ \\
\hline Intraoperative blood loss, mean $\pm \mathrm{SD}, \mathrm{mL}$ & $451.52 \pm 395.74$ \\
\hline
\end{tabular}

$\mathrm{SH}$, solid hemangioblastoma; VHL, von Hippel-Lindau.

was obtained at our institution. Basic patient characteristics including age, gender, tumor size, and tumor location were recorded; additionally, preoperative indicators including red blood cell (RBC) count, hemoglobin (HB), hematocrit (HCT), platelets (PLT), prothrombin time (PT), and activated partial thromboplastin time (APTT) were collected. From MRI, the diameter and location of the tumor were assessed, along with striking features including peritumoral edema, the flow-void effect, large draining veins, and the presence or absence of a visible feeding artery. The flow-void effect was defined by $\mathrm{T} 1$-weighted images and $\mathrm{T} 2$-weighted images; perifocal edema was defined by $\mathrm{T} 2$-weighted images. The identification of large draining veins and a feeding artery was established by operative video and surgical records, and then the distinctive characteristic of large draining veins and the feeding artery was confirmed in turn on MRI. Excessive IBL is consistent with the exhibition of large draining veins and a feeding artery on MRI. Also, large draining veins and a feeding artery could sometimes be defined by DSA. The volume of the IBL and the length of the operation time were available from the surgical records. The values and correlation of IBL and neuroimaging features were assessed by three professors-a practicing senior neurosurgeon and two neuroradiologists - who had more than 10 years of experience each. Large draining veins and feeding arteries were identified by intraoperative video and surgical records. Details on 4 unique MRI features identified in patients are shown in Figure 1.

In this study, IBL values were divided into three classes. Since an adult human has a blood volume of 4,000-5,000 mL, blood loss of $600 \mathrm{~mL}(15 \%)$ will cause class 1 shock (5). According to evidence from clinical practice, IBL less than $400 \mathrm{~mL}$ can safely be ignored; when the IBL is between 400 and $800 \mathrm{~mL}$, the surgeon should pay attention to the patient's condition; if the volume of IBL is $800 \mathrm{~mL}$ or more, there is a risk of shock. The patients were divided into the bleeding group and the control group according to IBL: if the amount of bleeding was $<400 \mathrm{~mL}$, and the amount of intraoperative bleeding was considered small, the patient was assigned to the control group; any patient who lost $\geq 400 \mathrm{~mL}$ or more was assigned to the bleeding group. The potential risk factors affecting IBL were explored in this study. Hence, we chose to convert the value of IBL into categorical covariates and explore the extent of the impact of risk factors upon IBL in SH.

\section{Statistical analysis}

Statistical analysis was performed using SPSS 22.0 software. The measurement data are expressed as the mean \pm SEM, and Mann-Whitney U-tests were used for comparison between groups. The count data are expressed as the rate (\%), and comparison between groups was performed using the $\chi^{2}$ test or Fisher's exact test. $\mathrm{P}<0.05$ was considered significant. The $\chi^{2}$ test, ordinal logistic regression, and Pearson correlation analysis were used to test relationships between variables. The significance level, $\alpha$, was set to 0.05 .

\section{Results}

The average size and IBL for SH in the present study were 
A
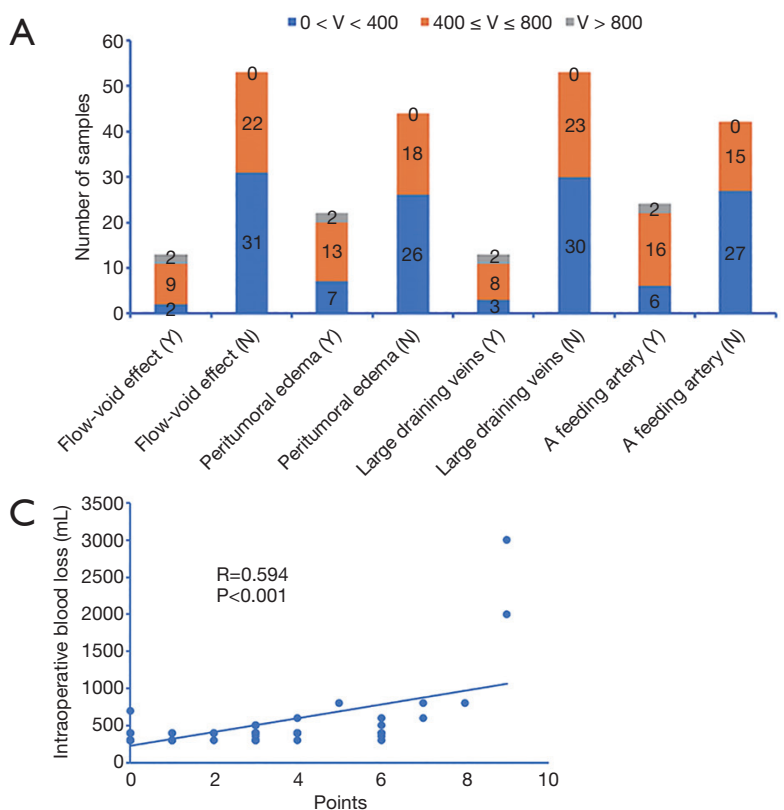

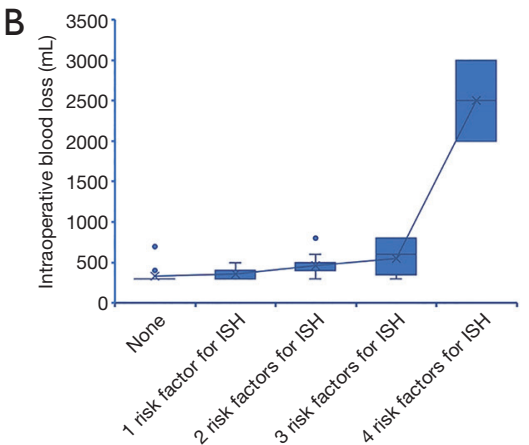

D

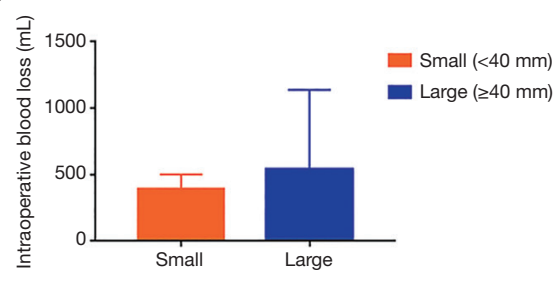

Figure 1 The relationship between factors and IBL. The distributions of four striking MRI features in patients with high-volume intraoperative blood loss (A); the tendency of more risk factors significantly contributing to disastrous intraoperative blood loss was established in the present study (B); the positive correlation between present scores and intraoperative blood loss (blue dots) was confirmed in this study $(\mathrm{R}=0.594)(\mathrm{C})$; greater IBL was observed from large SHs rather than in those with small diameters (D). Y, yes; N, no; MRI, magnetic resonance imaging; IBL, intraoperative blood loss; SH, solid hemangioblastoma.

$38.20 \pm 12.47 \mathrm{~mm}$ and $451.52 \pm 395.74 \mathrm{~mL}$, respectively. Fourteen patients were misdiagnosed as having medulloblastoma, ependymoma (Figure 2), or meningioma (Figure 3). The misdiagnosis rate in the present series was $21.2 \%$. Abundant IBL was observed in misdiagnosed cases due to unsuitable surgical strategies without en bloc resection. As for $\mathrm{SH}$ cases that were accurately diagnosed before surgery, less IBL was found in the preoperative embolism group than in the nonembolism group $(\mathrm{P}<0.05$; Figures 4,5$)$. The characteristics of neuroimaging showed the following high-risk factors for excessive IBL (Table 2): There were 11 patients with a flow-void effect on MRI in the bleeding group and 2 such patients in the control group; the difference was significant $(7.759, \mathrm{P}<0.05)$. The bleeding group contained 18 patients with obvious feeding arteries, whereas there were 6 cases in the control group; the incidence of feeding arteries was higher in the bleeding group than in the control group $(9.429, \mathrm{P}<0.05)$. Also, 10 cases in the bleeding group and 3 cases in the control group showed large drainage veins on MRI, meaning a significantly elevated occurrence of large drainage veins in the bleeding group compared with the control group (4.694,
$\mathrm{P}<0.05)$. Peritumoral edema was observed in 15 cases in the bleeding group and 7 cases in the control group, for a significant difference $(4.364, \mathrm{P}<0.05)$. The present findings show that several prominent MRI features are predictors of major IBL.

The weighted values of the high-risk factors in Table 3 showed remarkable IBL in further ordinal logistic regression and correlation analysis, which showed that the flow-void effect was the factor most significantly correlated with IBL $(\mathrm{P}<0.01, \mathrm{R}=0.418)$. The feeding artery also played a vital role in excessive IBL $(\mathrm{P}<0.05, \mathrm{R}=0.412)$. The next most influential factor was large draining veins $(\mathrm{P}<0.05, \mathrm{R}=0.350)$, followed by peritumoral edema $(\mathrm{P}<0.05$, $\mathrm{R}=0.308)$. The four major risk factors including the flowvoid effect, feeding arteries, large draining veins, and peritumoral edema were assigned point values of 3, 3, 2, and 1, respectively, for a largest total score of 9 points. The greater the total score for these four items in $\mathrm{SH}$, the higher the volume of bleeding and the greater the risk of major bleeding during surgery; the positive correlation between these scores and IBL was confirmed in this study (Figure 1). More interestingly, we found a positive relationship between 

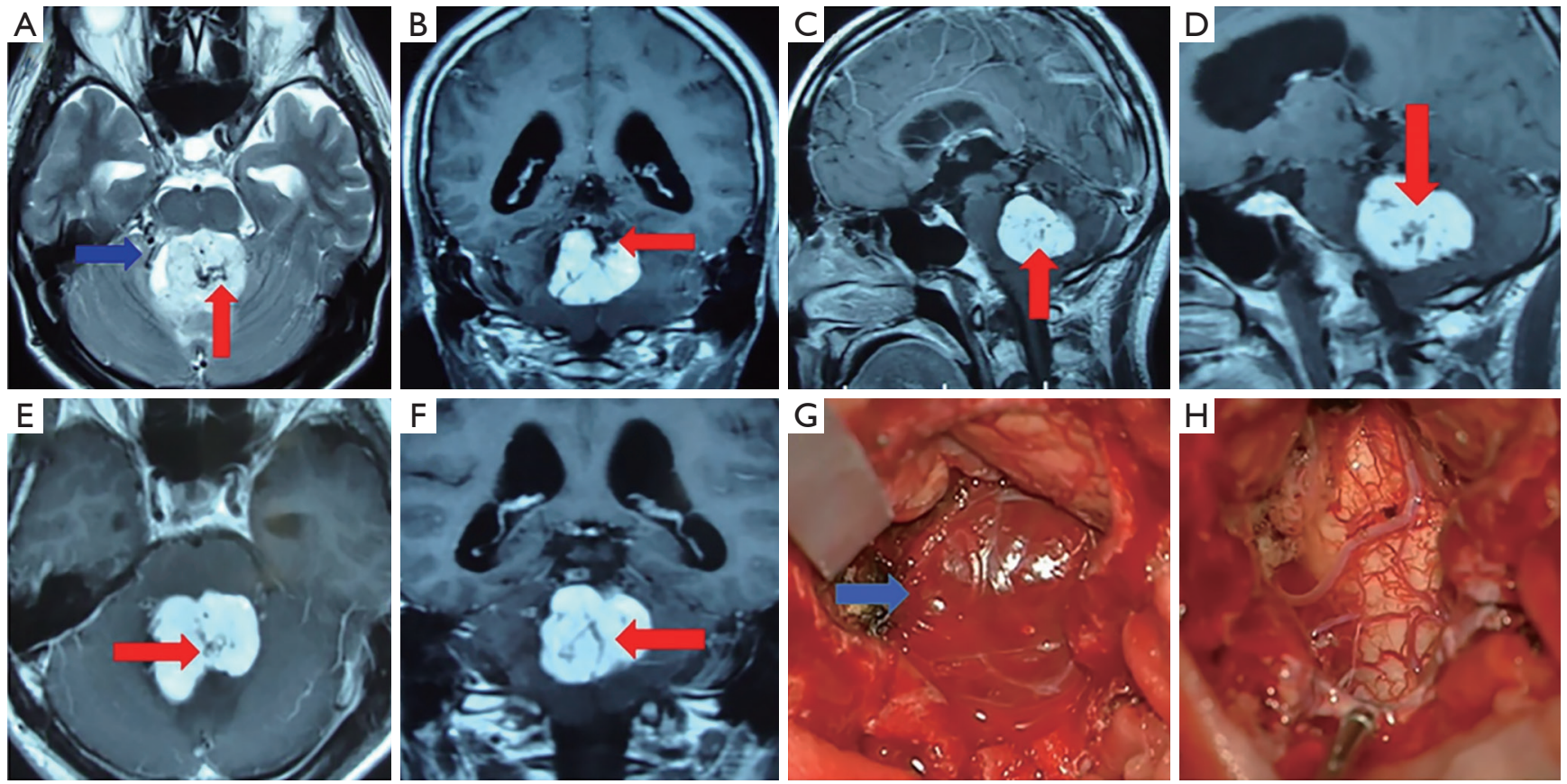

Figure 2 MRI scans showing abundant feeding arteries in the peritumoral region of the cerebellum (blue arrow) and intratumor structure (red arrow). Imaging studies revealed peritumor edema (A), large draining veins to the tentorium (B) and a marked flow-void effect (C,D,E,F); this patient was misdiagnosed as having medulloblastoma or ependymoma and did not undergo preoperative embolization. This tumor was confirmed as SH $(\mathrm{G})$ and was completely resected $(\mathrm{H})$. Intraoperative blood loss reached 3,000 $\mathrm{mL}$ in this case, and the scans indicated the presence of a flow-void effect (3 points), a feeding artery (3 points), large draining veins ( 2 points), and peritumoral edema (1 point). The sum of the scores was 9 points, which indicated that there was a high probability of massive blood loss during surgery. MRI, magnetic resonance imaging; $\mathrm{SH}$, solid hemangioblastoma.

the length of the operation and the total IBL $(\mathrm{P}<0.001)$. Moreover, though our analysis did not support a correlation between tumor size and IBL, interestingly, we found that IBL increased significantly when tumor diameter $\geq 40 \mathrm{~mm}$ $(\mathrm{P}<0.05$; Figure 1).

\section{Discussion}

Complete microsurgical removal of $\mathrm{SH}$ can be associated with increased risks due to the surrounding vital structures and the hypervascularity of this tumor type. $\mathrm{SH}$ is still one of the most challenging diseases for neurosurgeons due to catastrophic intraoperative bleeding. Young and Richardson (6) reported that death or a poor result occurred in $50 \%$ of 14 cases with $\mathrm{SH}$ of the posterior fossa. Subsequently, Dwarakanath et al. (7) reported 69 cases of microsurgery for hemangioblastoma at a single center; 8 deaths and 15 recurrences occurred in his study, for a mortality rate and a recurrence rate of $11 \%$ and $21 \%$, respectively. In 2012, Sakamoto et al. (8) reported 15 cases of hemangioblastoma; despite preoperative embolization of the tumor-supplying vessels in 7 of the 15 cases, 3 of those 7 patients had catastrophic intraoperative bleeding $(1,000 \mathrm{~mL})$, and there was 1 case of postoperative death, along with 1 case of moderate to severe disability. A recent publication reported an immediate postoperative mortality and morbidity of $7.8 \%$ and $17.2 \%$, respectively, for brainstem hemangioblastomas even when treated by an experienced neurosurgeon (9). Our study developed a grading standard based on imaging results. Four major risk factors, namely, the flow-void effect, feeding arteries, large draining veins, and the peritumoral edema, were scored as 3, 3, 2, and 1 according to their contribution to IBL, with the highest possible score being 9 points. The greater the sum of the four factors, the larger the amount of intraoperative bleeding and the greater the risk that it will occur. Moreover, patients with peritumoral edema had a high degree of postoperative edema. The 

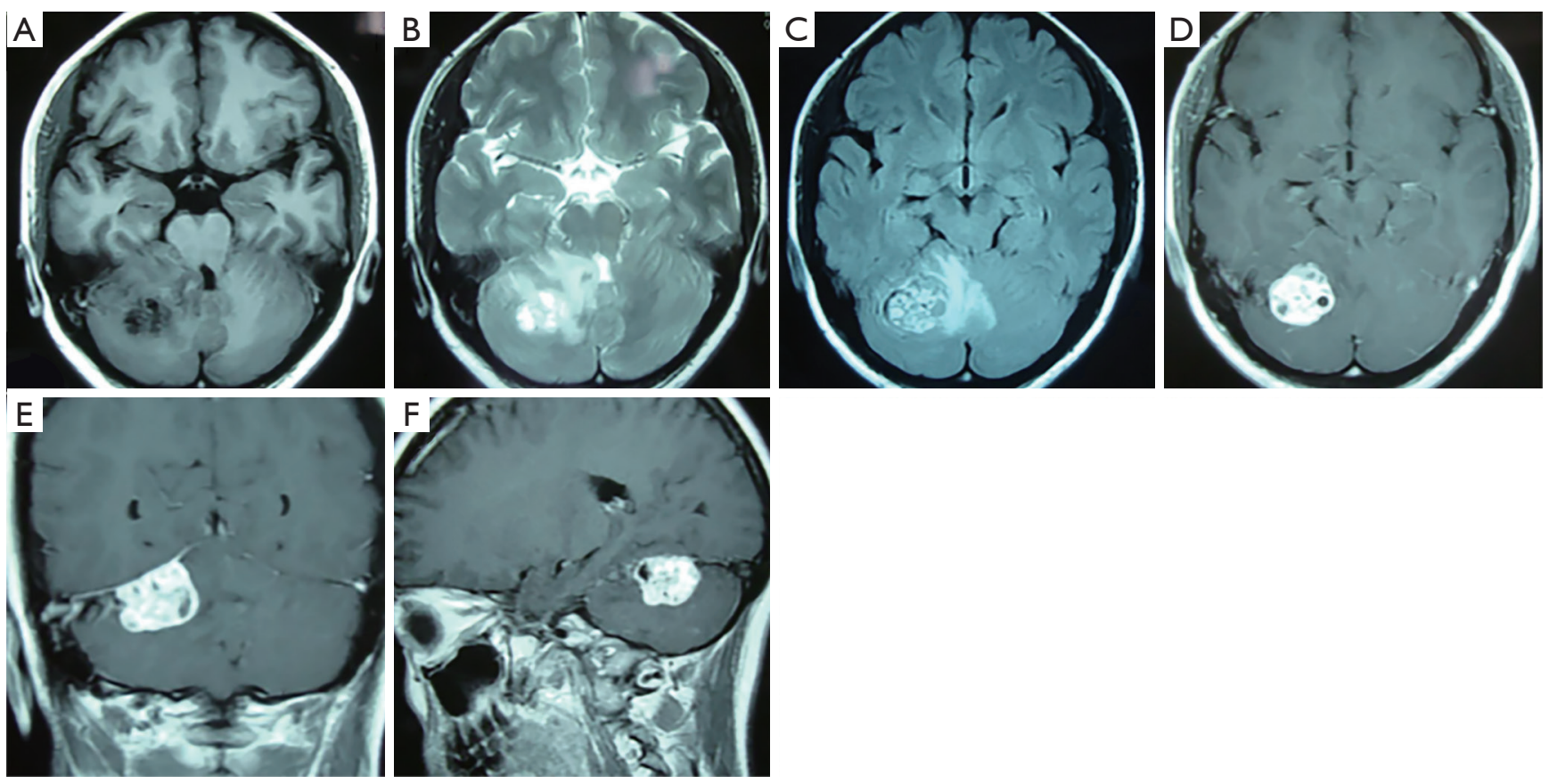

Figure $3 \mathrm{MRI}$ scans showing a lesion of the right cerebellum, adjacent to the tentorium, with brainstem compression and peritumoral edema (A,B,C), enhanced MRI showing that the lesion was linked to the tentorium and revealing a flow-void effect (D,E,F). The patient was misdiagnosed as having meningioma in the right cerebellopontine angle. The volume of intraoperative bleeding, in this case, was $600 \mathrm{~mL}$. Due to the absence of both a feeding artery ( 3 points) and large draining veins ( 2 points) on preoperative MRI, the total score was 4 points, consisting of 3 points for the flow-void effect and 1 point for peritumoral edema; this score predicts a low probability of major blood loss during surgery. MRI, magnetic resonance imaging.
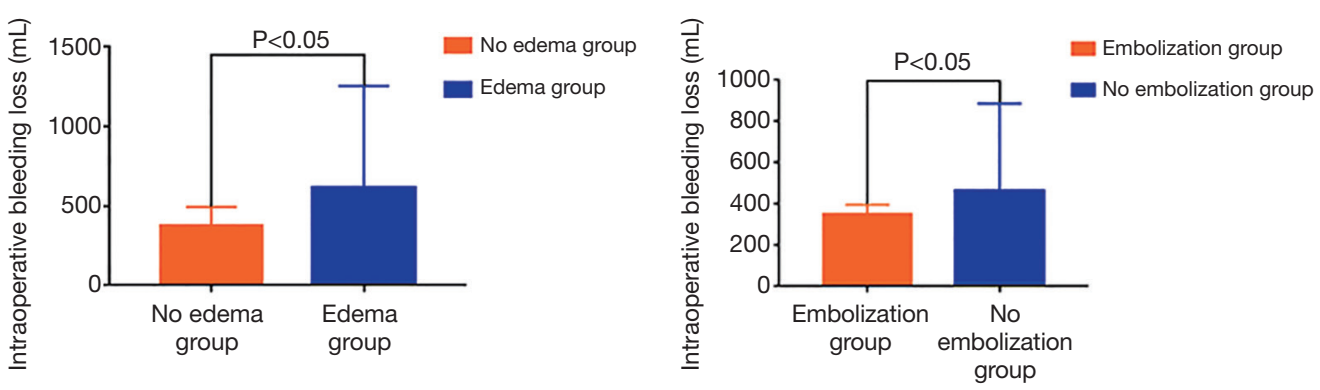

Figure 4 The left column graph shows that the volume of intraoperative blood loss was remarkably elevated in patients with peritumoral edema compared to those without peritumor edema; the right column graph shows that the volume of intraoperative blood loss was remarkably diminished in the embolization group compared to the nonembolization group.

study has important clinical value in determining the risk of intraoperative bleeding before surgical SH treatment, providing substantial guidance to surgeons so that they can take effective risk-reducing measures and avoid inappropriate surgical strategies such as piecemeal resection of SH.

The commonly used examination tools for preoperative imaging evaluation of SH include MRI, CTA, and DSA. Unfortunately, atypical SH was usually misdiagnosed as ependymoma or medulloblastoma or meningioma in our clinical practice based on MRI; some patients experienced severe bleeding and poor outcome due to piecemeal resection of $\mathrm{SH}$. Therefore, when the lesions involved in the dorsal brainstem and the cerebellum show the presence 

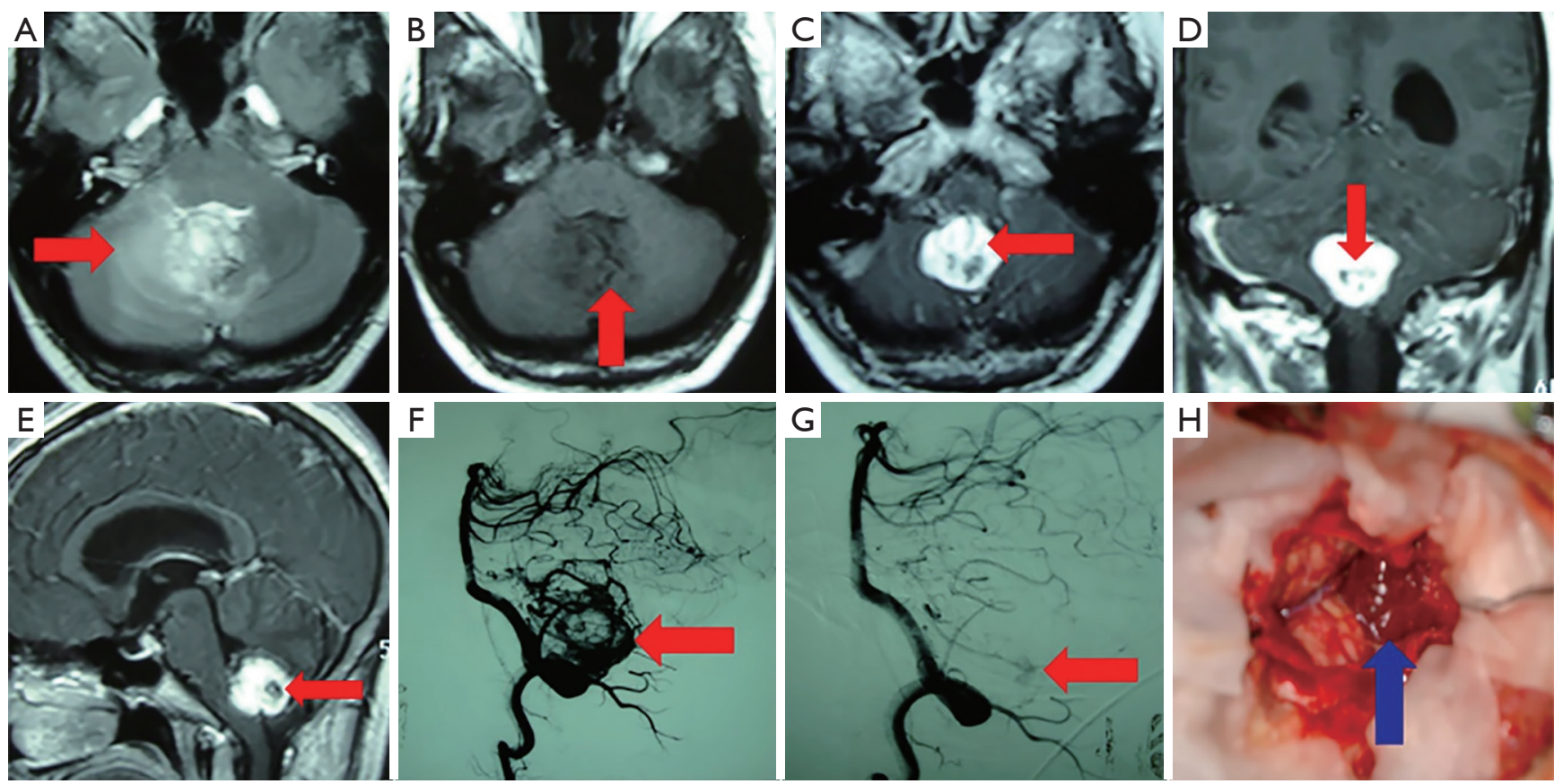

Figure 5 MRI scans (red arrow), DSA scans (red arrow) and intraoperative micrograph (blue arrow) showing the condition of this lesion in the cerebellum. MRI showing peritumoral edema (1 point) on T2-weighted images (A) and the flow-void effect (3 points) on T1-weighted images (B,C,D,E). Preoperative DSA revealed a feeding artery from the posterior inferior cerebellar artery (F) and a diminished blood supply after embolization $(\mathrm{G})$. An intraoperative micrograph is also shown $(\mathrm{H})$. The patient's total score was 4 points, which predicted that intraoperative bleeding would remain within an acceptable range. The intraoperative blood loss, in this case, was only $300 \mathrm{~mL}$. MRI, magnetic resonance imaging; DSA, digital subtraction angiography.

of a flow-void effect and the other abovementioned features indicate that the tumor has a large blood supply, $\mathrm{SH}$ should be highlighted, and it is wise to conduct CTA and DSA examinations upon diagnosis. The choice of CTA or DSA examination depends on the general condition of the patients, the Karnofsky Performance Status (KPS) score, and the wishes of the patient's family. If the patient has obstructive hydrocephalus and poor physical signs typical of elderly patients, safe and rapid CTA can often identify the blood vessels supplying the tumor, and the advantage of DSA examination is that it can reveal the blood supply of the tumor even during a surgical embolization intervention. From our analysis, DSA is effective and reliable in detecting diminished blood loss after embolization in this study. Additionally, the novel scoring system proposed by our group uses a combination of factors from clinical imaging data to predict intraoperative hemorrhage, marking that the first highrisk factors have been used to quantify the bleeding risk in surgery for intracranial SH. Our scale has strong predictive power and clinical practicability. According to this study, once patients with a high degree of suspicion of $\mathrm{SH}$ in the posterior fossa are confirmed, measures can be taken to help ensure the effectiveness of treatment. For example, in preoperative embolization and the choice of a suitable manner of removal, performing en bloc resection rather than a piecemeal resection can significantly reduce the amount of IBL and reduce the risk of disastrous bleeding. Of course, there is also some controversy concerning the preoperative embolization of hemangioblastoma; for example, Ene et al. (10) reported 24 cases of hemangioblastoma after embolization, 3 cases of bleeding, and 3 cases of infarction complications, with the incidence of permanent neurological impairment being $15 \%$. Therefore, preoperative embolization should be evaluated with care. In addition, Ampie et al. (11) asserted that preoperative embolization could not increase the total tumor resection rate and reduce the amount of IBL. Conversely, Rangel-Castilla et al. (12) reported that preoperative embolization of high-blood-supply tumors of the head, 
Table 2 Correlation factors of intraoperative blood loss in SH

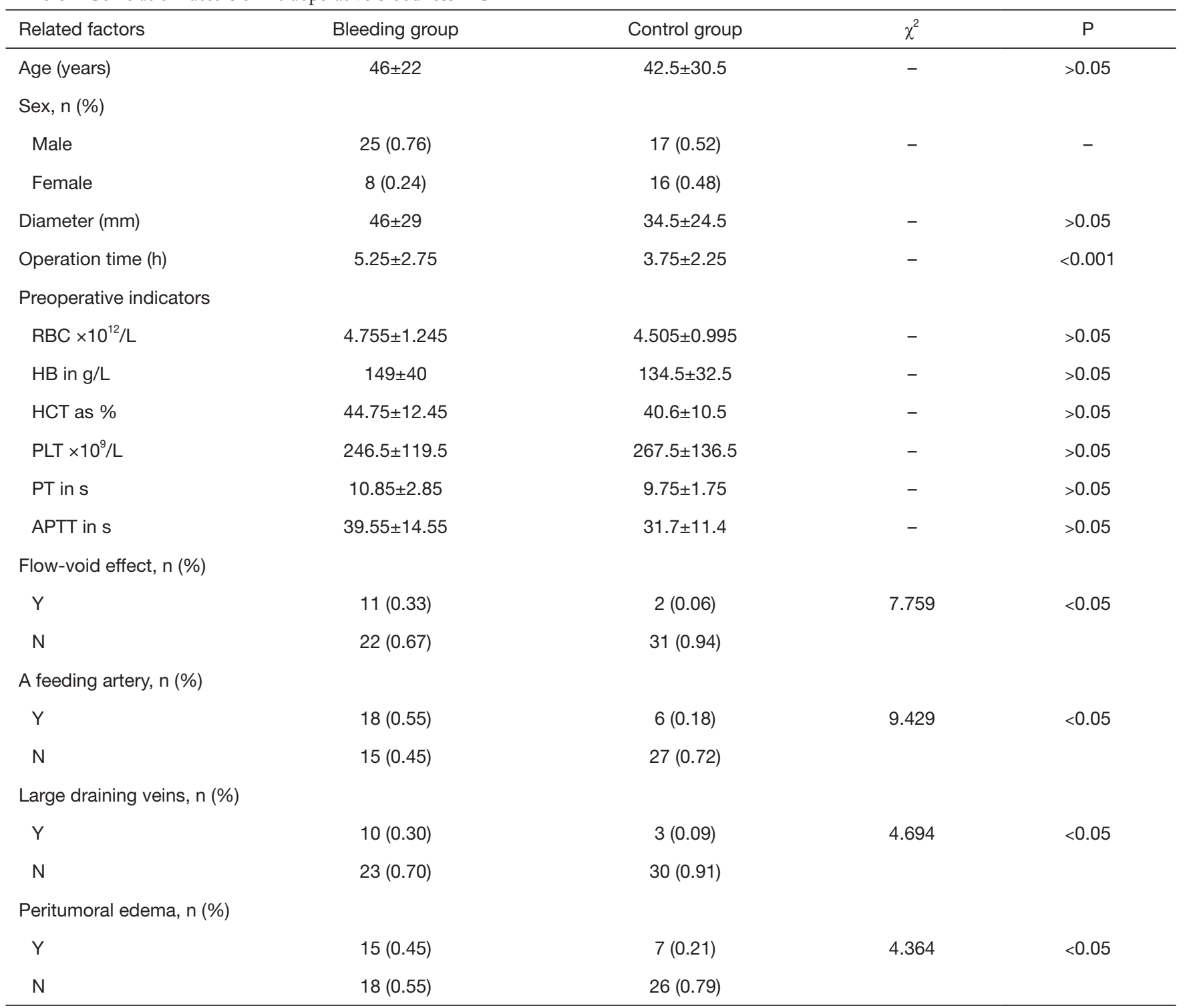

SH, solid hemangioblastoma; RBC, red blood cell; HB, hemoglobin; HCT, hematocrit; PLT, platelets; PT, prothrombin time; APTT, activated partial thromboplastin time; $\mathrm{Y}$, yes; N, no.

neck, and spinal cord was a safe and effective treatment; 100 tumor patients, including 3 with hemangioblastoma, underwent preoperative embolization, and none of them died or developed serious complications. Accumulating evidence has demonstrated that preoperative endovascular embolization is a safe and effective adjunct treatment that facilitates the safe and complete resection of SHs $(13,14)$. According to our experience, it is necessary to accurately embolize substantial hemangioblastomas that have a large blood supply and to actively cooperate with the interventional department before surgery to communicate with the main blood supply vessels. This measure is crucial in reducing the damage caused by embolization, especially embolization of deep blood vessels not easily exposed during operation rather than superficial blood supply arteries, which greatly benefits the success of the operation. We have performed a precise embolization on a patient with thrice-relapsed SH to achieve safe resection. However, patients undergoing preoperative investigations are usually kept as inpatients for several days. This, along with the 
Table 3 Results of ordinal logistic regression and correlation of risk factors of IBL predict by MRI in SH

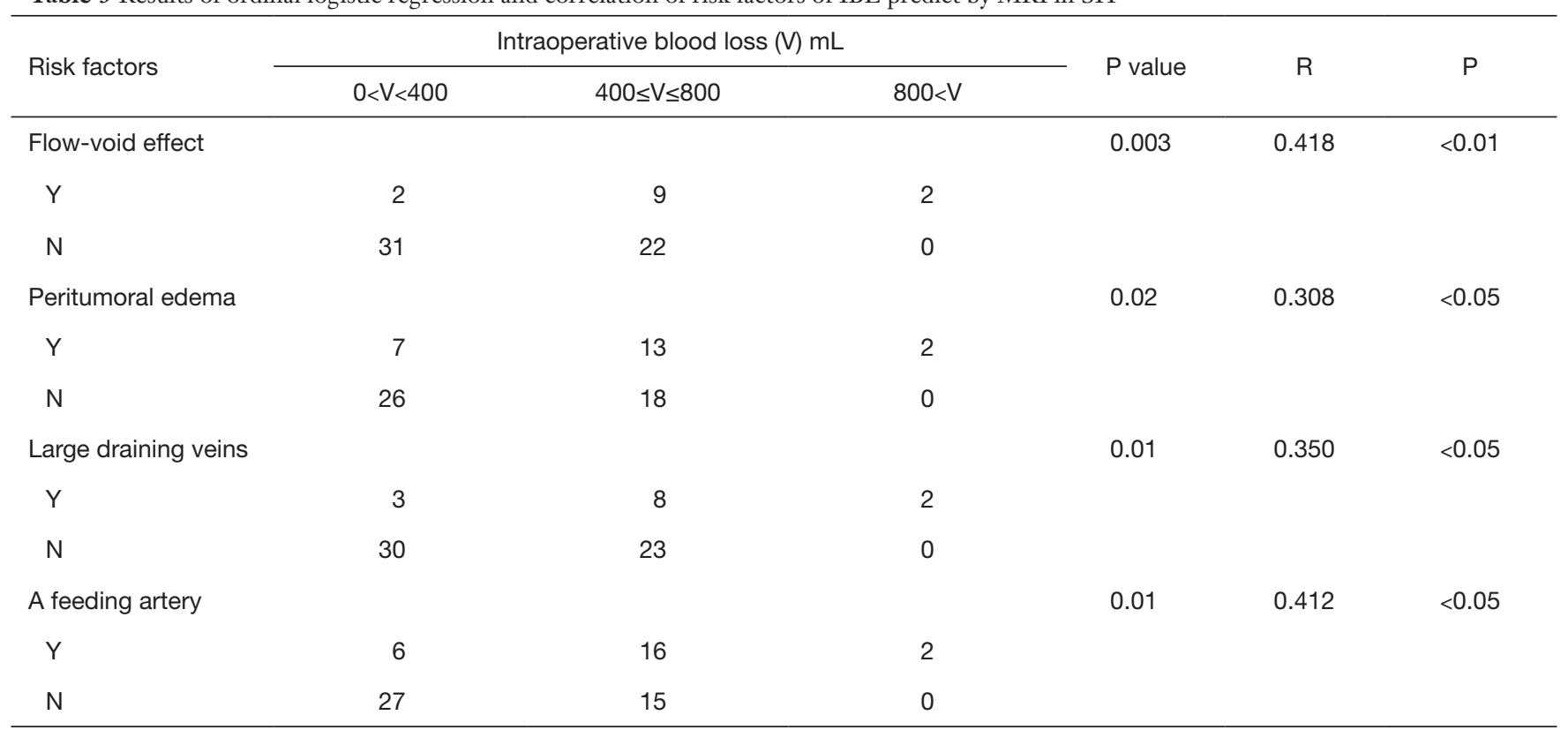

IBL, intraoperative blood loss; MRI, magnetic resonance imaging; $\mathrm{SH}$, solid hemangioblastoma; Y, yes; N, no.

presence of a brain tumor, increases the risk in developing deep venous thrombosis or pulmonary embolus, and may pose additional challenges to surgical resection, which thus necessitates the use of low-molecular-weight heparins (LMWH) capable of reducing the risk of thromboembolism before surgery (15). Meanwhile, Chibbaro et al. showed that preoperative $\mathrm{LMWH}$ does not increase such a risk of bleeding, but a sub-analysis was not done according to the degree of the vascularity of tumors. Hence, the clinical dilemma of whether to administer LMWH or not for SH has not yet been resolved (16). Moreover, the literature reports that stereotactic radiotherapy for multiple small yet substantial hemangioblastomas associated with VHL is an effective treatment. Kano et al. (17) reported that the 1 -, 3-, and 5-year progression-free survival of stereotactic radiotherapy for hemangioblastoma was $96.9 \%, 95 \%$, and $89.9 \%$, respectively. The authors asserted that stereotactic radiotherapy has a higher tumor control rate and weaker radiotherapy side effects than conventional radiotherapy and constitutes an important adjuvant treatment for hemangioblastoma. Notably, the safety of stereotactic radiosurgery for the management of these posterior fossa lesions is increasing in light of the ongoing development of radio-enhancers that may unlock the potential of radiation therapy within tumor cells while sparing the surrounding healthy brain parenchyma (18). In the future, these aspects will likely transform into more tailored theranostic strategies (19).

Recently, Lagman et al. (20) reported that the flow-void effect can be used as an indicator of IBL in meningiomas without embolization. The Meningioma Vascularity Index (MVI) was calculated by measuring flow-void volumes on T2-weighted MR images. The study revealed an interesting phenomenon: intraoperative estimated blood loss in nonembolized meningiomas was correlated with MVI, and an increased MVI was associated with increased risks of blood transfusion and subtotal resection. However, these relationships were not found in the embolized group. Our study revealed the same tendency: the presence of the flowvoid effect indicating, to some extent, an increase in IBL when we performed tumor resection. Also, other unique features of MRI, such as obvious feeding arteries, large draining veins, and peritumoral edema were shown for the first time to be associated with excessive IBL.

This study had some limitations. First, the 66 consecutive patients with $\mathrm{SH}$ received their surgery from several different senior professors rather than a single experienced neurosurgeon; discrepancies in neurosurgical skill may affect IBL. Second, analysis bias of MRI features by three radiologists might have had a significant confounding effect on the prediction of IBL. Finally, only 66 cases were included in the present study; a larger sample of SH patients 
is needed in future research.

The present work is the first to report the potential neuroimaging-assessed risk factors leading to abundant IBL in SH. We discovered that four MRI features, namely, the flow-void effect, feeding arteries, large draining veins, and peritumoral edema, were risk factors closely related to excessive IBL in SH. Second, this study is the first to develop a scoring system based on high-risk factors for intracranial SH to predict IBL. Our novel scoring system for predicting excessive IBL is the first such system proposed and will significantly contribute to decreasing the surgical risk of SH treatment.

\section{Acknowledgments}

None.

\section{Footnote}

Conflicts of Interest: The authors have no conflicts of interest to declare.

Ethical Statement: The present study was approved by the ethics committee of Zhengzhou University in accordance with the Declaration of Helsinki. All patients gave written informed consent for the surgery and for the use of their data and samples for research purposes before the surgery.

\section{References}

1. Takayanagi S, Mukasa A, Tanaka S, Nomura M, Omata M, Yanagisawa S, Ichimura K, Nakatomi H, Ueki K, Aburatani H, Saito N. Differences in genetic and epigenetic alterations between von Hippel-Lindau diseaserelated and sporadic hemangioblastomas of the central nervous system. Neuro Oncol 2017;19:1228-36.

2 Kuharic M, Jankovic D, Splavski B, Boop FA, Arnautovic KI. Hemangioblastomas of the Posterior Cranial Fossa in Adults: Demographics, Clinical, Morphologic, Pathologic, Surgical Features, and Outcomes. A Systematic Review. World Neurosurg 2018;110:e1049-62.

3 Kageji T, Nagahiro S, Mizobuchi Y, Nakajima K. Postoperative Hematoma Requiring Recraniotomy in 1149 Consecutive Patients With Intracranial Tumors. Oper Neurosurg (Hagerstown) 2017;13:392-7.

4 Tabibkhooei A, Fattahi A, Rahatlou H. Presentation of a Hemangioblastoma in cavernous sinus: An extremely rare case report. Int J Surg Case Rep 2018;44:54-6.
5 Spaniol JR, Knight R, Zebley JL, Anderson D, Pierce JD. Fluid resuscitation therapy for hemorrhagic shock. J Trauma Nurs 2007;14:152-60.

6 Young S, Richardson AE. Solid haemangioblastomas of the posterior fossa: radiological features and results of surgery. J Neurol Neurosurg Psychiatry 1987;50:155-8.

7 Dwarakanath S, Suri A, Sharma BS, Mehta VS. Intracranial hemangioblastomas: an institutional experience. Neurol India 2006;54:276-8.

8 Sakamoto N, Ishikawa E, Nakai Y, Akutsu H, Yamamoto T, Nakai K, Shiigai M, Tsurushima H, Isobe T, Takano S, Tsuboi K, Matsumura A. Preoperative endovascular embolization for hemangioblastoma in the posterior fossa. Neurol Med Chir (Tokyo) 2012;52:878-84.

9 Ma D, Wang Y, Du G, Zhou L. Neurosurgical Management of Brainstem Hemangioblastomas: A Single-Institution Experience with 116 Patients. World Neurosurg 2015;84:1030-8.

10 Ene CI, Xu D, Morton RP, Emerson S, Levitt MR, Barber J, Rostomily RC, Ghodke BV, Hallam DK, Albuquerque FC, McDougall CG, Sekhar LN, Ferreira M, Kim LJ, Chang SW. Safety and Efficacy of Preoperative Embolization of Intracranial Hemangioblastomas. Oper Neurosurg (Hagerstown) 2016;12:135-40.

11 Ampie L, Choy W, Lamano JB, Kesavabhotla K, Kaur R, Parsa AT, Bloch O. Safety and outcomes of preoperative embolization of intracranial hemangioblastomas: A systematic review. Clin Neurol Neurosurg 2016;150:143-51.

12 Rangel-Castilla L, Shah AH, Klucznik RP, Diaz OM. Preoperative Onyx embolization of hypervascular head, neck, and spinal tumors: experience with 100 consecutive cases from a single tertiary center. J Neurointerv Surg 2014;6:51-6.

13 Nair BR, Joseph V, Chacko G, Keshava SN. Giant solid hemangioblastoma of the cerebellopontine angle: a technically challenging case. Neurol India 2014;62:228-9.

14 Wu P, Liang C, Wang Y, Guo Z, Li B, Qiu B, Li X, Wen Z, Pan Q. Microneurosurgery in combination with endovascular embolisation in the treatment of solid haemangioblastoma in the dorsal medulla oblongata. Clin Neurol Neurosurg 2013;115:651-7.

15 Ganau M, Prisco L, Cebula H, Todeschi J, Abid H, Ligarotti G, Pop R, Proust F, Chibbaro S. Risk of Deep vein thrombosis in neurosurgery: State of the art on prophylaxis protocols and best clinical practices. J Clin Neurosci 2017;45:60-6.

16 Chibbaro S, Cebula H, Todeschi J, Fricia M, Vigouroux 
D, Abid H, Kourbanhoussen H, Pop R, Nannavecchia

B, Gubian A, Prisco L, Ligarotti GKI, Proust F, Ganau

M. Evolution of Prophylaxis Protocols for Venous

Thromboembolism in Neurosurgery: Results from a

Prospective Comparative Study on Low-Molecular-

Weight Heparin, Elastic Stockings, and Intermittent

Pneumatic Compression Devices. World Neurosurg 2018;109:e510-6.

17 Kano H, Niranjan A, Mongia S, Kondziolka D, Flickinger JC, Lunsford LD. The role of stereotactic radiosurgery for intracranial hemangioblastomas. Neurosurgery 2008;63:443-50.

Cite this article as: Gao Q, Zhang L, Wang G, Xu D, Feng M, Wang F, Wei Q, Liu X, Guo F. A novel neuroimaging assessment of predictive risk factors associated with intraoperative massive hemorrhage in intracranial solid hemangioblastoma. Quant Imaging Med Surg 2019;9(11):1781-1791. doi: 10.21037/qims.2019.09.05
18 M Ganau, RI Foroni, M Gerosa, GK Ricciardi, M Longhi, A Nicolato. Radiosurgical options in neuro-oncology: A review on current tenets and future opportunities. Part II: Adjuvant radiobiological tools. Tumori 2015;101:57-63.

19 Min KH, Lee HJ, Lee SC, Park K. Biomineralized hybrid nanoparticles for imaging and therapy of cancers. Quant Imaging Med Surg. 2018;8:694-708.

20 Lagman C, Ong V, Nguyen T, Alkhalid Y, Sheppard JP, Romiyo P, Azzam D, Prashant GN, Jahan R, Yang I. The Meningioma Vascularity Index: a volumetric analysis of flow voids to predict intraoperative blood loss in nonembolized meningiomas. J Neurosurg 2018;1:1-6. 